\title{
Correction to: Association Between Temporal Spatial Parameters and Overuse Injury History in Runners: A Systematic Review and Meta-analysis
}

\author{
Richard A. Brindle ${ }^{1}$ (I) $\cdot$ Jeffrey B. Taylor ${ }^{1} \cdot$ Coty Rajek $^{1} \cdot$ Anika Weisbrod $^{2} \cdot$ Kevin R. Ford $^{1}$
}

Published online: 19 November 2019

(c) Springer Nature Switzerland AG 2019

Corrections to: Sports Medicine https://doi.org/10.1007/s40279-019-01207-5

Page 1, Abstract, Results section, lines 17-20: The following sentence, which previously read:

"Healthy runners and those with a history of overuse injury had a similar average stride time (mean difference: $-0.01 \mathrm{~s}$, 95\% CI -0.02 to $0.01 \mathrm{~s}$ ), contact time (mean difference: $0.00 \mathrm{~s}, 95 \%$ CI $0.00-0.01 \mathrm{~s}$ ), cadence (mean difference: 0.3 steps per minute (spm), $95 \% \mathrm{CI}-1.8$ to $2.5 \mathrm{spm}$ ), and stride length (mean difference $0.00 \mathrm{~m}, 95 \% \mathrm{CI}-0.04$ to $0.05 \mathrm{~m}$ ) during running".

Should read:

"Healthy runners and those with a history of overuse injury had a similar average stride time (mean difference: $0.00 \mathrm{~s}$, 95\% CI -0.01 to $0.01 \mathrm{~s}$ ), contact time (mean difference: $0.00 \mathrm{~s}, 95 \% \mathrm{CI} 0.00$ to $0.01 \mathrm{~s}$ ), cadence (mean difference: 0.3 steps per minute (spm), $95 \% \mathrm{CI}-1.8$ to $2.5 \mathrm{spm}$ ), and stride length (mean difference $0.00 \mathrm{~m}, 95 \% \mathrm{CI}-0.05$ to $0.05 \mathrm{~m}$ ) during running".

The original article can be found online at https://doi.org/10.1007/ s40279-019-01207-5.

Richard A. Brindle

richard_brindle@baylor.edu

1 Department of Physical Therapy, High Point University, High Point, North Carolina, USA

2 Department of Exercise Science, High Point University, High Point, North Carolina, USA 
Page 4, Figure 1: The text in the 'Records excluded' box, which previously read 'Records excluded $(\mathrm{n}=1888)$ ':

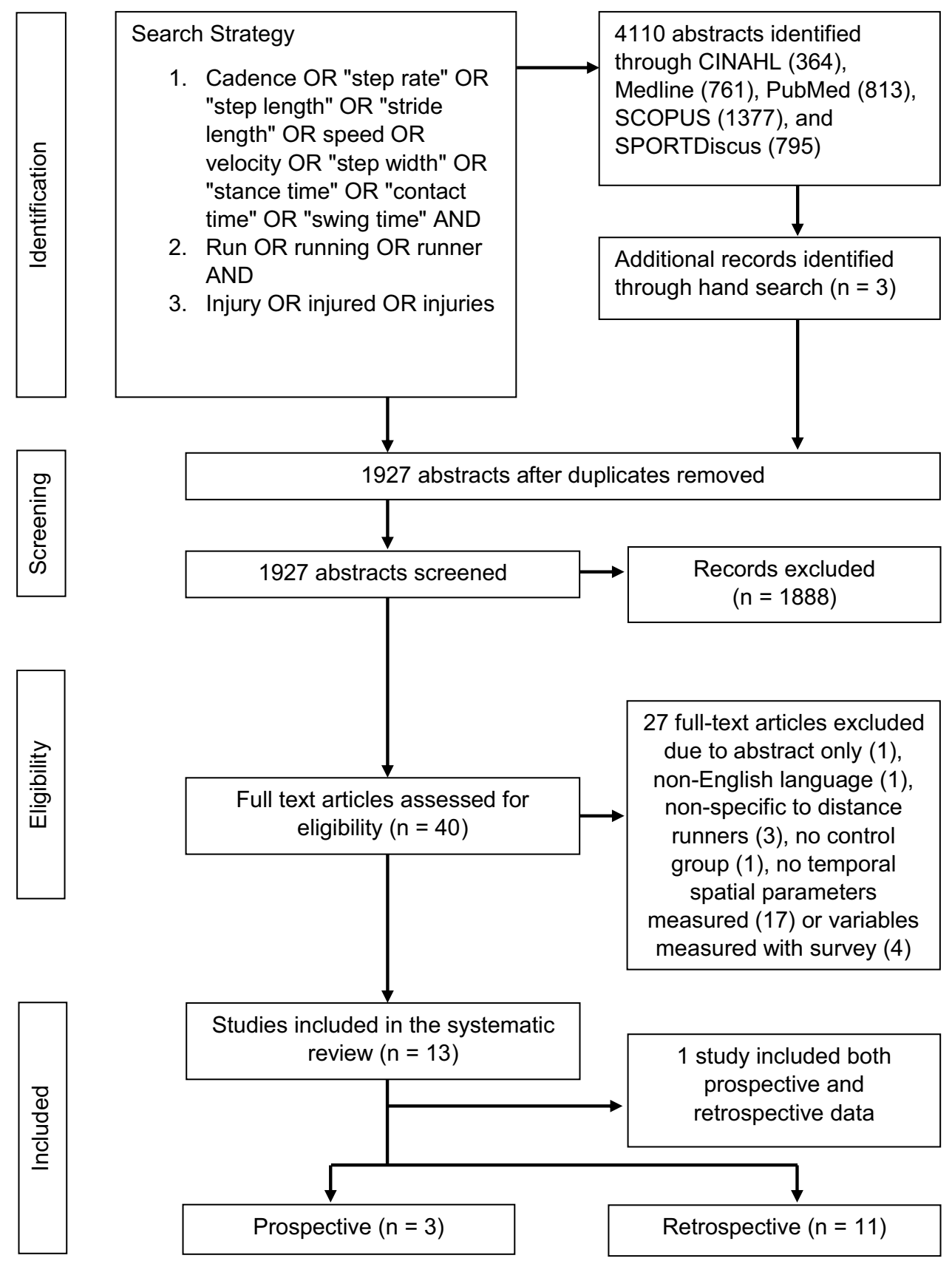


Should read: 'Records excluded $(\mathrm{n}=1887)$ '.

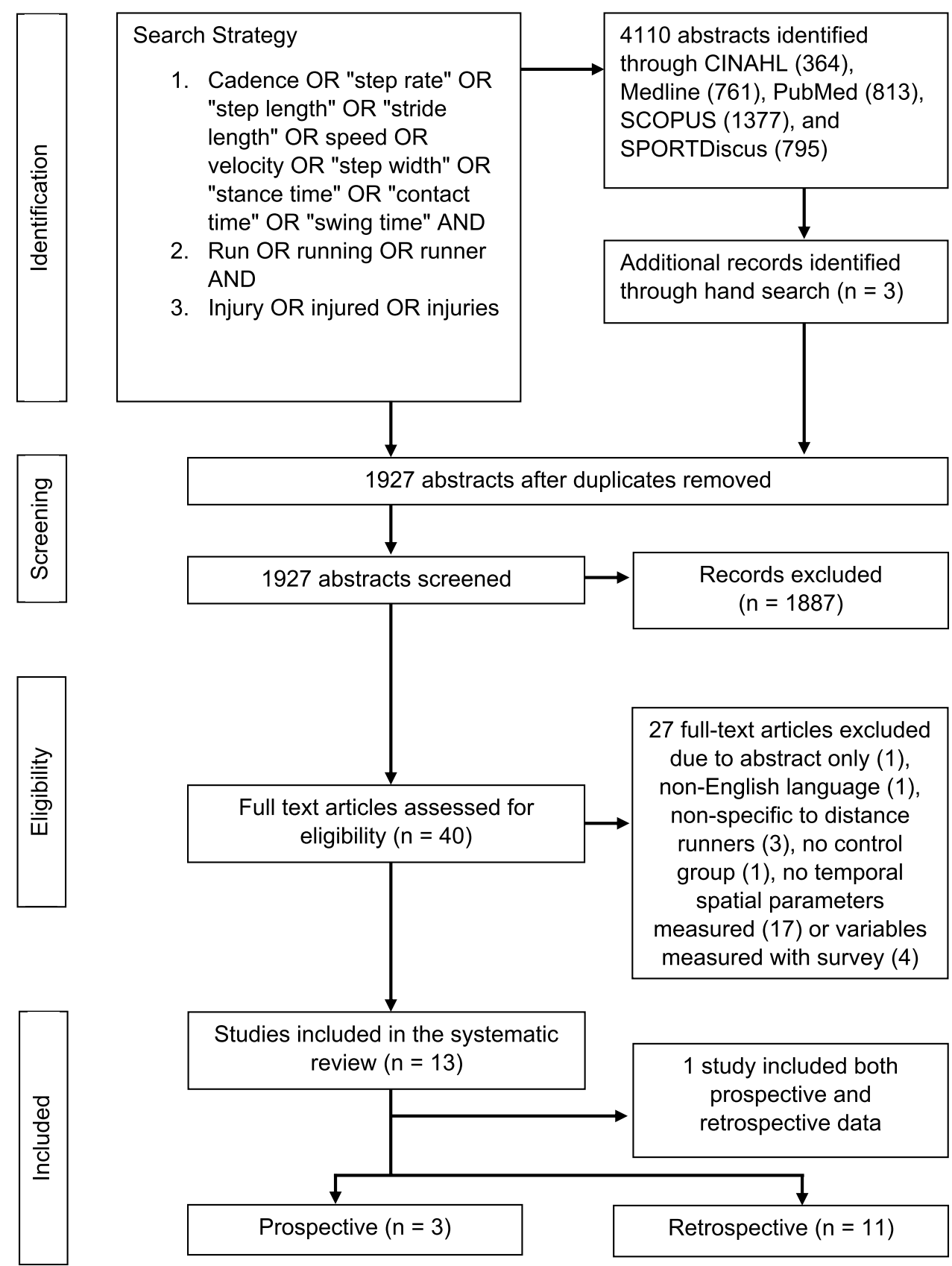

Page 6, Section 3.2, column 1, lines 6-9: The following sentence, which previously read:

"Stride time and stride length meta-analyses were composed of 8 and 10 group comparisons and data from 224 and 229 individuals, respectively".
Should read:

"Stride time and stride length meta-analyses were composed of 8 and 9 group comparisons and data from 224 and 206 individuals, respectively". 
Page 6, Section 3.2, column 1, lines 15-22: The following sentence, which previously read:

"Meta-analyses of stride time (mean difference: $0.00 \mathrm{~s}, 95 \%$ $\mathrm{CI}-0.01$ to $0.01 \mathrm{~s}$ ), contact time (mean difference: $0.00 \mathrm{~s}$, 95\% CI 0.00-0.01 s), cadence (mean difference: 0.3 steps per minute (spm), $95 \% \mathrm{CI}-1.8$ to $2.4 \mathrm{spm}$ ), and stride length (mean difference $0.00 \mathrm{~m}, 95 \% \mathrm{CI}-0.04$ to $0.05 \mathrm{~m}$ ) during running revealed no differences between the pooled injured and uninjured groups of runners ( $p>0.05$ ) (Figs. 2, 3, 4, 5)".

Should read:

"Meta-analyses of stride time (mean difference: $0.00 \mathrm{~s}, 95 \%$ $\mathrm{CI}-0.01$ to $0.01 \mathrm{~s}$ ), contact time (mean difference: $0.00 \mathrm{~s}$, 95\% CI 0.00 to $0.01 \mathrm{~s}$ ), cadence (mean difference: 0.3 steps per minute [spm], $95 \% \mathrm{CI}-1.8$ to $2.4 \mathrm{spm}$ ), and stride length (mean difference $0.00 \mathrm{~m}, 95 \% \mathrm{CI}-0.05$ to $0.05 \mathrm{~m}$ ) during running revealed no differences between the pooled injured and uninjured groups of runners ( $p>0.05$ ) (Figs. 2, 3, 4, 5)".

Page 7, Table 2, Prospective design studies section, Bredeweg et al. [20] row. The entry in the 'Injury' column, which previously read:

"Lower extremity injury in last 3 months".

Should read:

"Injury to lower extremity or back".

Page 7, Table 2, Prospective design studies section, Bredeweg et al. [19] row. The entry in the 'Injury' column, which previously read:
"Lower extremity injury in last 3 months".

Should read:

"Injury to lower extremity or back".

Page 7, Table 2, Cross-sectional design studies, Willson et al. [30] row. The entry in the 'TSP variables' column, which previously read:

"Stride length, contact time".

Should read:

"Step length, contact time".

Page 8, column 2, Section 4.4, lines 3-5: The following sentence, which previously read:

"Stride length during running was similar between injured and uninjured groups of runners in all studies included in this meta-analysis [23, 26, 29, 30]".

Should read:

"Stride length during running was similar between injured and uninjured groups of runners in all studies included in this meta-analysis [23, 26, 29]".

Page 9, Figure 2: The following figure, which previously read:

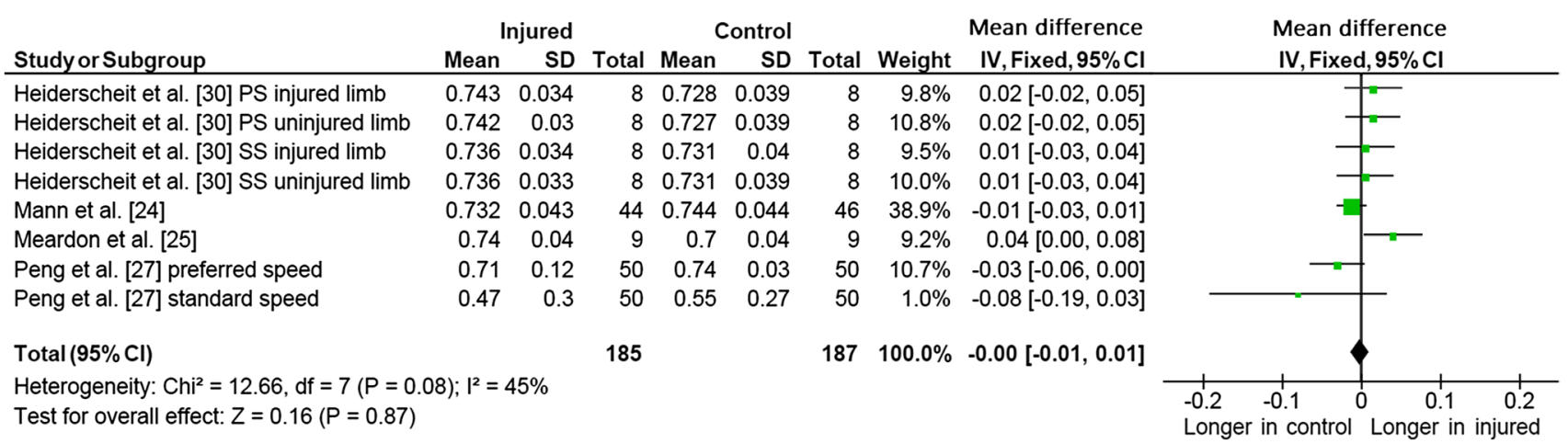


Should read:

\begin{tabular}{|c|c|c|c|c|c|c|c|c|c|}
\hline \multirow[b]{2}{*}{ Study or Subgroup } & \multicolumn{3}{|c|}{ Injured } & \multicolumn{3}{|c|}{ Control } & \multicolumn{2}{|r|}{ Mean difference } & \multirow{2}{*}{$\begin{array}{l}\text { Mean difference } \\
\text { IV, Fixed, } 95 \% \mathrm{Cl}\end{array}$} \\
\hline & Mean & SD & Total & Mean & SD & Total & Weight & IV, Fixed, $95 \% \mathrm{Cl}$ & \\
\hline Heiderscheit et al. [29] PS injured limb & 0.743 & 0.03 & 8 & 0.728 & 0.039 & 8 & $10.7 \%$ & $0.02[-0.02,0.05]$ & \\
\hline Heiderscheit et al. [29] PS uninjured limb & 0.742 & 0.03 & 8 & 0.727 & 0.039 & 8 & $10.7 \%$ & $0.02[-0.02,0.05]$ & \\
\hline Heiderscheit et al. [29] SS injured limb & 0.736 & 0.034 & 8 & 0.731 & 0.04 & 8 & $9.4 \%$ & $0.01[-0.03,0.04]$ & \\
\hline Heiderscheit et al. [29] SS uninjured limb & 0.736 & 0.033 & 8 & 0.731 & 0.039 & 8 & $9.9 \%$ & $0.01[-0.03,0.04]$ & \\
\hline Mann et al. [23] & 0.732 & 0.043 & 44 & 0.744 & 0.044 & 46 & $38.5 \%$ & $-0.01[-0.03,0.01]$ & \\
\hline Meardon et al. [24] & 0.74 & 0.04 & 9 & 0.7 & 0.04 & 9 & $9.1 \%$ & $0.04[0.00,0.08]$ & \\
\hline Peng et al. [26] preferred speed & 0.71 & 0.12 & 50 & 0.74 & 0.03 & 50 & $10.6 \%$ & $-0.03[-0.06,0.00]$ & \\
\hline Peng et al. [26] standard speed & 0.47 & 0.3 & 50 & 0.55 & 0.27 & 50 & $1.0 \%$ & $-0.08[-0.19,0.03]$ & \\
\hline Total $(95 \% \mathrm{Cl})$ & & & 185 & & & 187 & $100.0 \%$ & $-0.00[-0.01,0.01]$ & \\
\hline \multicolumn{4}{|c|}{$\begin{array}{l}\text { Heterogeneity: } \text { Chi }^{2}=12.74, d f=7(P=0.08) ; I^{2}=45 \% \\
\text { Test for overall effect: } Z=0.14(P=0.89)\end{array}$} & & & & & & $\begin{array}{lllll}-0.2 & -0.1 & 0 & 0.1 & 0.2 \\
\text { Longer in control } & \text { Longer in injured }\end{array}$ \\
\hline
\end{tabular}

Page 9, Figure 2: The following figure caption, which previously read:

"Fig. 2 Forest plot of stride time (s) during running".

Should read:
"Fig. 2 Forest plot of stride time (s) during running. $S D$ standard deviation, $I V$ inverse variance, $C I$ confidence interval, $P S$ preferred speed, $S S$ standard speed".

Page 9, Figure 3: The following figure, which previously read:

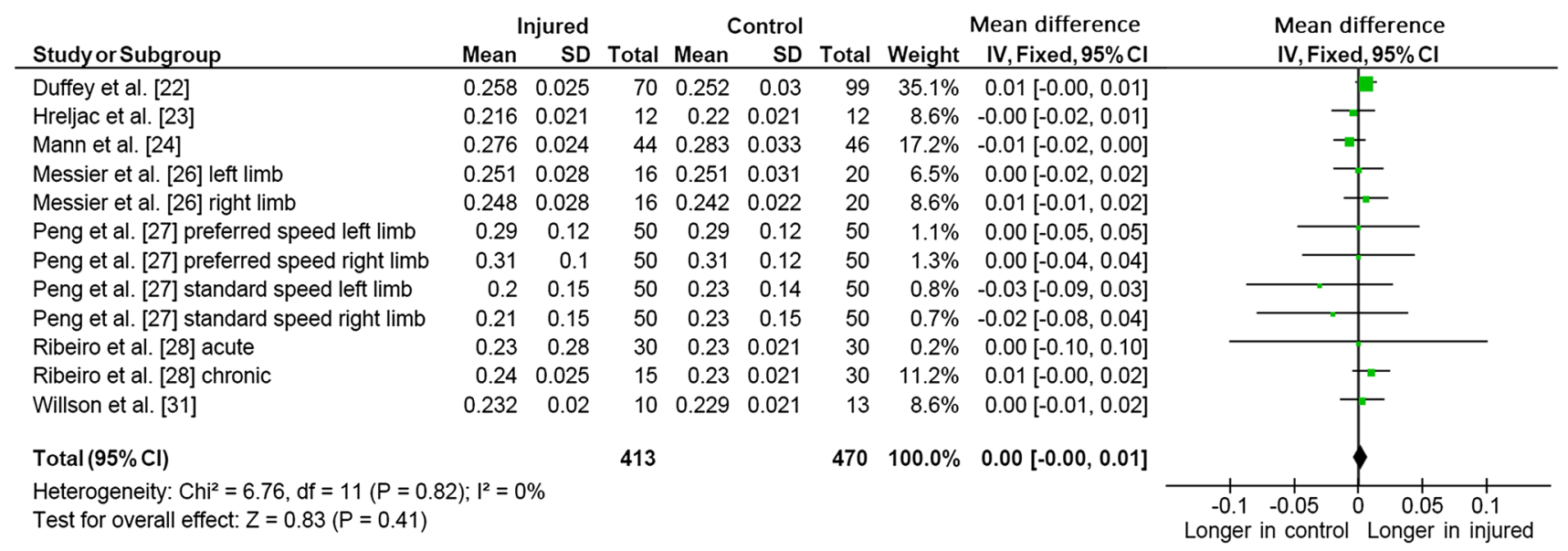


Should read:

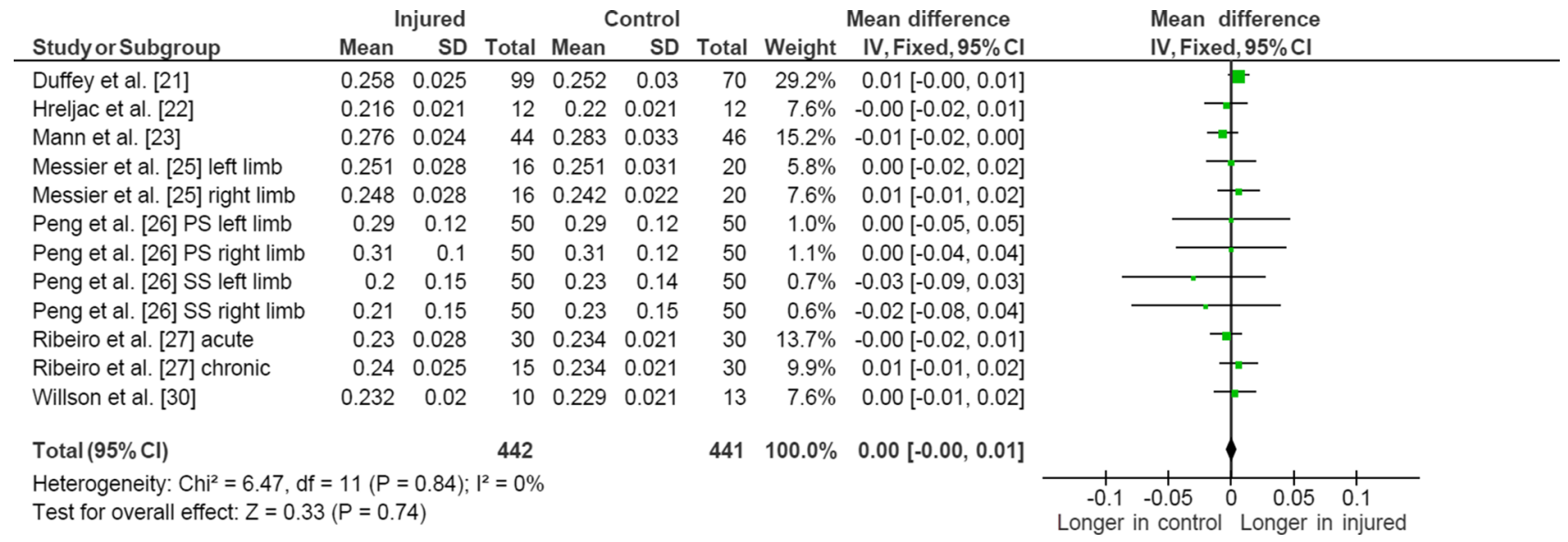

Page 9, Figure 3: The following figure caption, which previously read:

"Fig. 3 Forest plot of contact time (s) during running".

Should read:
"Fig. 3 Forest plot of contact time (s) during running. $S D$ standard deviation, $I V$ inverse variance, $C I$ confidence interval, $P S$ preferred speed, $S S$ standard speed".

Page 9, Figure 4: The following figure, which previously read:

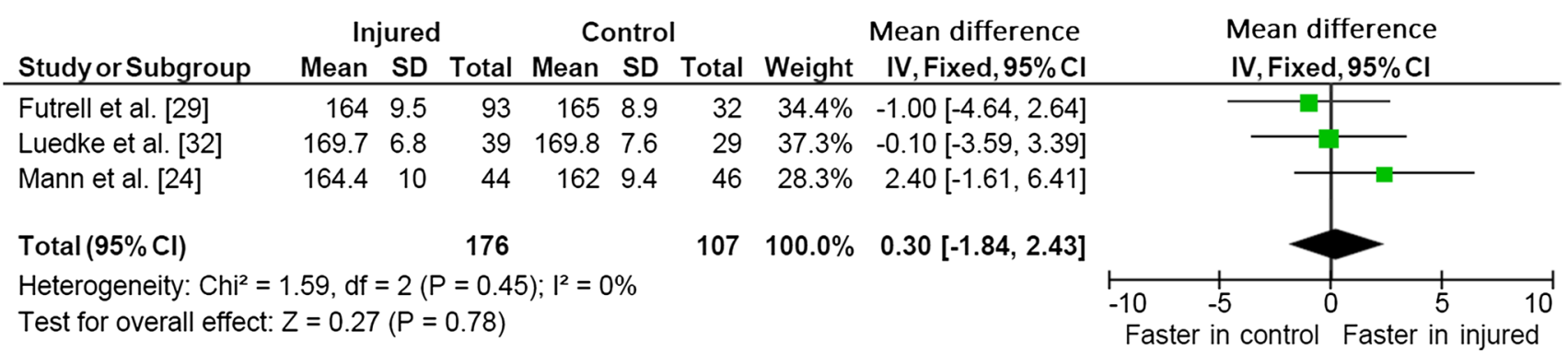

Should read:

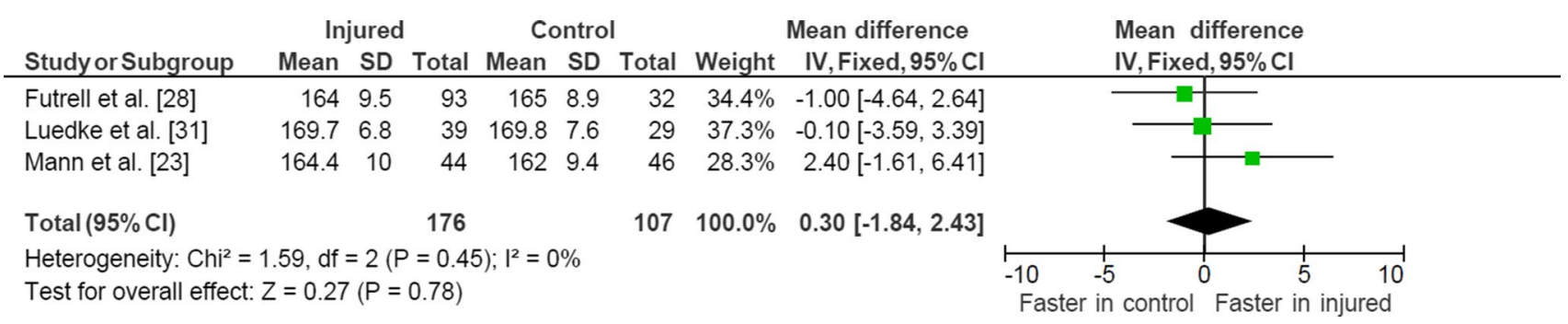


Page 9, Figure 4: The following figure caption, which previously read:

"Fig. 4 Forest plot of cadence (steps per minute) during running".

Should read:

"Fig. 4 Forest plot of cadence (steps per minute) during running. $S D$ standard deviation, $I V$ inverse variance, $C I$ confidence interval".

Page 10, Figure 5: The following figure, which previously read:
Page 10, Figure 5: The following figure caption, which previously read:

"Fig. 5 Forest plot of stride length (m) during running".

Should read:

"Fig. 5 Forest plot of stride length (m) during running. $S D$ standard deviation, $I V$ inverse variance, $C I$ confidence interval, $P S$ preferred speed, $S S$ standard speed".

The original article has been corrected.

\begin{tabular}{|c|c|c|c|c|c|c|c|c|c|c|}
\hline \multirow[b]{2}{*}{ Study or Subgroup } & \multicolumn{3}{|c|}{ Injured } & \multicolumn{3}{|c|}{ Control } & \multicolumn{2}{|r|}{ Mean difference } & \multirow{2}{*}{\multicolumn{2}{|c|}{$\begin{array}{l}\text { Mean difference } \\
\text { IV,Fixed, } 95 \% \mathrm{Cl}\end{array}$}} \\
\hline & Mean & SD & Total & Mean & SD & Total & Weight & IV, Fixed, $95 \% \mathrm{Cl}$ & & \\
\hline Heiderscheit et al. [30] PS injured limb & 1.92 & 0.22 & 8 & 1.98 & 0.17 & 8 & $3.5 \%$ & $-0.06[-0.25,0.13]$ & & \\
\hline Heiderscheit et al. [30] PS uninjured limb & 1.91 & 0.22 & 8 & 1.98 & 0.17 & 8 & $3.5 \%$ & $-0.07[-0.26,0.12]$ & & \\
\hline Heiderscheit et al. [30] SS injured limb & 1.98 & 0.09 & 8 & 1.97 & 0.12 & 8 & $11.9 \%$ & $0.01[-0.09,0.11]$ & & \\
\hline Heiderscheit et al. [30] SS uninjured limb & 1.98 & 0.09 & 8 & 1.97 & 0.12 & 8 & $11.9 \%$ & $0.01[-0.09,0.11]$ & & \\
\hline Mann et al. [24] & 2.205 & 0.32 & 44 & 2.174 & 0.323 & 46 & $7.3 \%$ & $0.03[-0.10,0.16]$ & & \\
\hline Peng et al. [27] PS left limb & 1.53 & 0.52 & 50 & 1.47 & 0.63 & 50 & $2.5 \%$ & $0.06[-0.17,0.29]$ & & \\
\hline Peng et al. [27] PS right limb & 1.53 & 0.52 & 50 & 1.47 & 0.63 & 50 & $2.5 \%$ & $0.06[-0.17,0.29]$ & & \\
\hline Peng et al. [27] SS left limb & 1.6 & 1.14 & 50 & 1.82 & 1.13 & 50 & $0.6 \%$ & $-0.22[-0.66,0.22]$ & & \\
\hline Peng et al. [27] SS right limb & 1.59 & 1.14 & 50 & 1.82 & 1.13 & 50 & $0.6 \%$ & $-0.23[-0.67,0.21]$ & & \\
\hline Willson et al. [31] & 1.463 & 0.054 & 10 & 1.459 & 0.063 & 13 & $55.8 \%$ & $0.00[-0.04,0.05]$ & & \\
\hline Total $(95 \% \mathrm{Cl})$ & & & 286 & & & 291 & $100.0 \%$ & $0.00[-0.03,0.04]$ & & \\
\hline $\begin{array}{l}\text { Heterogeneity: } \mathrm{Chi}^{2}=3.67, \mathrm{df}=9(P=0.93 \\
\text { Test for overall effect: } Z=0.13(P=0.89)\end{array}$ & $1^{2}=0$ & & & & & & & & $\begin{array}{lll} & 1 & 1 \\
-1 & -0.5 & 0 \\
& \text { Longer in control Longer }\end{array}$ & $\begin{array}{l}0.5 \\
\text { in injured }\end{array}$ \\
\hline
\end{tabular}

Should read:

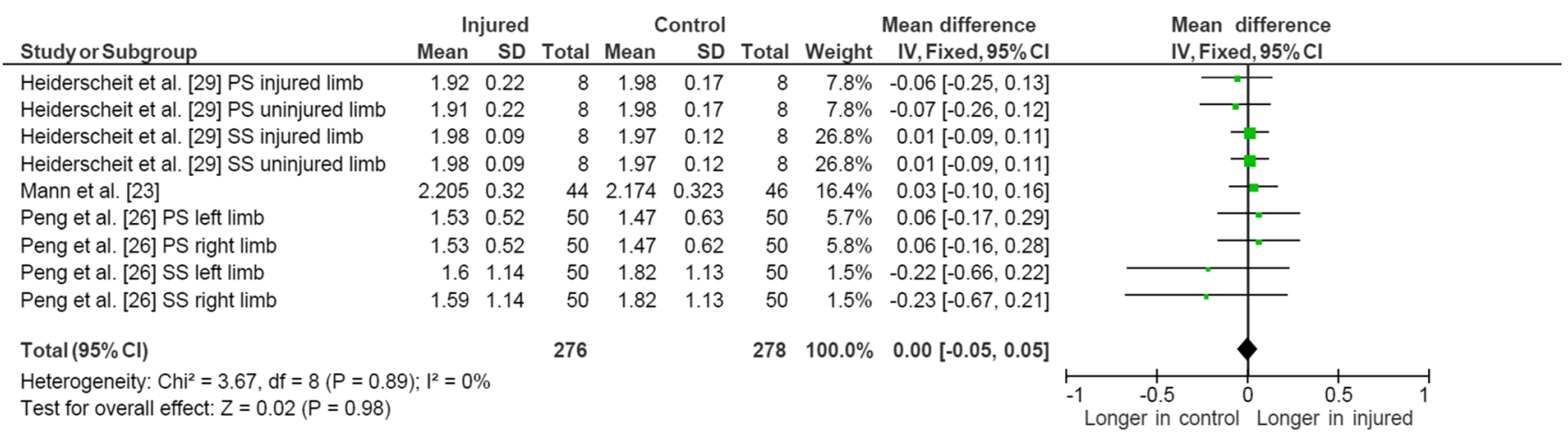

